\title{
Hierarchical organization as a determinant of sequential learning
}

\author{
HARVEY H. C. MARMUREK \\ University of Guelph, Guelph, Ontario, Canada \\ and \\ NEAL F. JOHNSON \\ Ohio State University, Columbus, Ohio 43210
}

\begin{abstract}
Subjects learned a set of permutations of a base sequence of letters. A set of permutations either defined a hierarchical organization for the base sequence or did not. Sets that defined organizations led to more correct responses, and the pattern of interitem sequential dependencies revealed that subjects had learned the organization defined by a response set. Differences in learning could not be explained in terms of the frequency with which items occurred adjacently because that frequency was held constant for both organization-defining and organization-free response sets. The difficulty of learning a particular organization was related to the memory load induced by the organization, and those differences were more consistent with a model of sequential learning proposed by Johnson (1970) than they were with a model proposed by Estes (1972).
\end{abstract}

When Lashley (1951) identified the problem that the serial nature of behavior provided for associative chain theory, he noted that psychologists had neglected the organizational processes underlying behavior. Recently, however, cognitive psychologists have increased their use of serial learning tasks to study those organizational processes (Bower, 1970; Martin $\&$ Noreen, 1974). The general hypothesis guiding those studies is that a learner encodes a long sequence as a series of subgroupings. Support for that hypothesis has been provided by demonstrations of recognition decrements (Bower \& Winzenz, 1969) and retroactive inhibition (Johnson \& Migdoll, 1971) after a change in the grouping of items within a sequence.

Two questions arise from the idea that a sequence is stored in the form of subgroupings: (1) What is the relationship of the elements in a sequence to the subgroupings, and (2) what is the relationship among the subgroupings? Theories to account for these relationships have been proposed by Estes (1972) and Johnson (1970). According to both of these theories, individual elements in the sequence which form a particular subgrouping are represented by a common abstract code. The subgroupings are represented by yet higher level codes, with the result that the entire sequence is represented as a hierarchy of codes.

Estes (1972) and Johnson (1970) propose different mechanisms to account for the retention of order

This paper is based on a thesis submitted by the first author to Ohio State University in partial fulfillment of the requirements for the master's degree. Requests for reprints should be sent to Harvey H. C. Marmurek, Department of Psychology, University of Guelph, Guelph, Ontario, Canada. information when a sequence of items is learned. According to Johnson (1970) a hierarchical organization of codes is decoded in a top-to-bottom left-to-right fashion. To begin the sequence, the learner retrieves the highest level code and decodes it into its subordinate codes. The rightmost code is then stored in memory, and the leftmost code is decoded further. This process continues until an individual element in the sequence is produced. At that point, the code most recently stored in memory is retrieved and decoded. These decoding operations continue until all the individual elements (or as many as the learner can retrieve) are produced.

According to Johnson's theory, hierarchical structures may be quantified by assigning to each transition in the sequence the number of decoding operations required to produce the elements in the highest order code which begins with the element immediately following the transition. Consider, for example, the right-imbalanced structure in Figure 1. For the first transition, the letter $B$ is the immediately
RIGHT-IMBALANCED

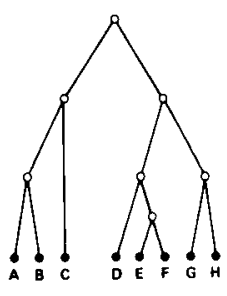

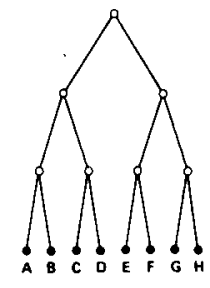

BALANCED

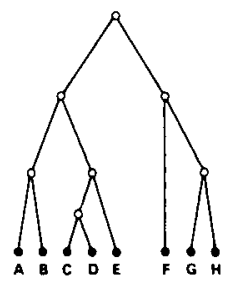

LEFT-IMEALANCED
Figure 1. Hierarchical organizations defined by the consistent permutation sets. 
following element. The highest order code beginning with $B$ is the code representing $B$, and only one operation is required to decode that code into the letter B. For the third transition, however, the letter $D$ is the immediately following element. The highest order code beginning with $\mathrm{D}$ is the code that represents DEFGH. The number of operations required to produce the elements represented by that code is determined as follows: (1) two operations to decode the DEFGH code into DEF and GH codes; (2) two operations to decode the DEF code into D and EF codes; (3) one operation to decode the $\mathrm{D}$ code into the letter $\mathrm{D}$; (4) two operations to decode the EF code in to E and F codes; (5) two operations to decode the $E$ and $F$ codes into the letters $E$ and $F$; (6) two operations to decode the $\mathrm{GH}$ code into $\mathrm{G}$ and $\mathrm{H}$ codes; and (7) two operations to decode the $G$ and $H$ codes into the letters $G$ and $H$. Thus, the structural value assigned to Transitions 1 and 3 are 1 and 13, respectively. The structural values, in terms of decoding operations, assigned to each transition in the structures shown in Figure 1 are listed in Table 1.

Estes (1972) proposed that sequential order information is preserved in a hierarchical system through the process of rehearsal. According to Estes (1972, p. 183) the production of an ordered sequence entails the inhibition of terminal elements until the output of earlier items in the sequence. Consequently, Estes (1972) assumes that the representation of ordered information comprises both associative and inhibitory connections. As items are rehearsed, excitatory connections are formed between control elements and the lower level items associated to them. At each level in the hierarchy, inhibitory connections occur laterally among the elements sharing a common control element. The calculation of inhibitory connections assumes that the items represented by the lowest nodes in each structure are rehearsed in either a uniform left-to-right or a uniform right-to-left order (Estes, Note 1). The inhibitory connections are crucial in determining the sequential output of elements in that an item's position of output is inversely related to the number of inhibitory connections to that item (see Estes, 1972, p. 185).
Consider again the right-imbalanced structure in Figure 1. Associative connections are represented by the line segments projecting downward from one node to another; there are 14 of them. One inhibitory connection is counted for each pair of segments projecting downward from the same node; there are seven inhibitory connections in the right-imbalanced structure. The number of associative (14) and inhibitory (7) connections is the same for all three structures shown in Figure 1.

In learning a sequence of elements, a subject must learn two sorts of information: the items in the sequence and the positions of those items within the sequence. Healy (1974) has shown that item and order information entail different processes, but she studied only short strings of letters and did not manipulate structural complexity. Since the Estes (1972) and Johnson (1970) models differ in their accounts of how order information is maintained, the present study was designed to study the learning of order information only. To isolate the ordering process, the subjects were provided with the to-be-learned items on each trial. The task for the subjects was to learn a set of permutations of those items that either did or did not define a hierarchical organization for the sequence that was permuted.

Keeney (1969) has shown that certain sets of permutations will lead subjects to learn an implicit structuring of the elements that are permuted. The rule for selecting the permutations is that only those permutations that assign elements to unique nodes in the structure may be used. For example, consider the sequence $A B C D$ and the hierarchical structure ((AB) (CD)). According to Keeney (1969), the permutation $C D A B$ defines $A B$ and $C D$ as two subgroupings of $A B C D$ since only elements that are represented by unique nodes may be permuted. The permutation BACD defines $\mathrm{A}$ and $\mathrm{B}$ and lower order constituents of $A B$ and the permutation $A B D C$ defines $C$ and $D$ as constituents of $C D$. To support the structureinducing property of the technique, Keeney (1969) showed that subjects who learned one set of systemdefining permutations and then transferred to a list of new letters that retained the old system produced

Table 1

Mean TEP Values and Their Correlations with Decoding Operations

\begin{tabular}{|c|c|c|c|c|c|c|c|c|c|c|c|}
\hline \multirow[b]{2}{*}{ Condition } & & \multicolumn{7}{|c|}{ Transition } & \multirow[b]{2}{*}{ I } & \multirow[b]{2}{*}{$t(5)$} & \multirow[b]{2}{*}{$\mathbf{p}$} \\
\hline & & 1 & 2 & 3 & 4 & 5 & 6 & 7 & & & \\
\hline Balanced & $\begin{array}{l}\text { Decoding Operations } \\
\text { Consistent TEP } \\
\text { Inconsistent TEP }\end{array}$ & $\begin{array}{r}1 \\
.01 \\
.19\end{array}$ & $\begin{array}{r}4.09 \\
.30\end{array}$ & $\begin{array}{r}1 \\
.00 \\
.17\end{array}$ & $\begin{array}{r}10 \\
.19 \\
.49\end{array}$ & $\begin{array}{l}1 \\
.01 \\
.16\end{array}$ & $\begin{array}{r}4 \\
.03 \\
.19\end{array}$ & $\begin{array}{r}1 \\
.00 \\
.04\end{array}$ & $\begin{array}{l}.96 \\
.91\end{array}$ & $\begin{array}{l}8.4 \\
4.9\end{array}$ & $\begin{array}{l}<.001 \\
<.005\end{array}$ \\
\hline Right Branching & $\begin{array}{l}\text { Decoding Operations } \\
\text { Consistent TEP } \\
\text { Inconsistent TEP }\end{array}$ & $\begin{array}{r}1 \\
.03 \\
.12\end{array}$ & $\begin{array}{r}1 \\
.06 \\
.41\end{array}$ & $\begin{array}{r}13 \\
.24 \\
.36\end{array}$ & $\begin{array}{r}4 \\
.12 \\
.34\end{array}$ & $\begin{array}{l}1 \\
.08 \\
.32\end{array}$ & $\begin{array}{l}4 \\
.05 \\
.24\end{array}$ & $\begin{array}{l}1 \\
.01 \\
.05\end{array}$ & $\begin{array}{l}.92 \\
.37\end{array}$ & $\begin{array}{r}5.3 \\
.9\end{array}$ & $\begin{array}{l}<.01 \\
>.4\end{array}$ \\
\hline Left Branching & $\begin{array}{l}\text { Decoding Operations } \\
\text { Consistent TEP } \\
\text { Inconsistent TEP }\end{array}$ & $\begin{array}{l}1 \\
.04 \\
.10\end{array}$ & $\begin{array}{l}7 \\
.16 \\
.36\end{array}$ & $\begin{array}{r}4 \\
.13 \\
.31\end{array}$ & $\begin{array}{l}1 \\
.11 \\
.21\end{array}$ & $\begin{array}{l}7 \\
.15 \\
.21\end{array}$ & $\begin{array}{l}4 \\
.11 \\
.16\end{array}$ & $\begin{array}{r}1 \\
.05 \\
.26\end{array}$ & $\begin{array}{l}.86 \\
.48\end{array}$ & $\begin{array}{l}3.8 \\
1.2\end{array}$ & $\begin{array}{l}<.01 \\
>.25\end{array}$ \\
\hline
\end{tabular}


fewer errors than subjects who transferred to permutations defining a new system. Furthermore, the pausal patterns of subjects learning different response systems were judged to vary in accordance with the particular system learned.

The permutations used in the present study were selected to define one of three structures and for each of these sets a comparison set was devised in which no system was defined. That is, the comparison set would assign individual elements to more than one subgrouping. For example, a permutation of $A B C D$ such as $B A C D$ would assign $A$ and $B$ to a common node, whereas BCDA would assign $A$ and $B$ to different nodes. Thus, BACD and BCDA are inconsistent in their assignment of $A$ to a node in the hierarchy. If order information is stored in the nodes of a hierarchy, then the consistent permutation sets that assign elements to unique nodes should be learned more quickly than inconsistent permutation sets.

A test of the Estes (1972) and Johnson (1970) coding theories requires the demonstration that the subjects did learn the structures defined by the consistent permutation sets. Both theories predict that the retrieval of elements represented by a common code are dependent upon activation of that code, and therefore, those items should display a greater dependency in recall than elements represented by different codes. Recall dependency may be determined by computing transitional error probabilities (TEPs). The TEP is a measure of the conditional probability that an element in a sequence is recalled incorrectly given that the preceding element is recalled correctly. According to coding models of sequence learning, TEP values should be high at transitions between elements represented by (Johnson, 1970) or associated with (Estes, 1972) different codes.

It is possible that TEP values may be determined by interitem contiguity, although the coding views deny this. To test this possibility, the number of times each pair of different elements occurred adjacently in the set of permutations was equated across consistent and inconsistent sets. If learning the TEP patterns is determined by interitem contiguity, then the consistent and inconsistent sets should not lead to differences in the dependent measures. On the other hand, if order information is determined by organizational structures, then the sets should differ.

Another purpose of the present study was to test the Estes (1972) and Johnson (1970) models as descriptions of how order information is preserved when a sequence is learned. The basis of the test was to evaluate the metrics of complexity derived from the models as predictors of learning difficulty. The structures used in the present study (see Figure 1) are equal in terms of inhibitory connections (the Estes metric for serial order information), yet vary in terms of total number of decoding operations (the Johnson metric). Thus, whereas the Estes model predicts the structures would be of equal difficulty, the Johnson model predicts the structures would be learned at different rates.

The present study, then, had three goals: (1) to determine whether a structure-defining response set would be easier to learn than an inconsistent set; (2) to determine whether the influence of the structure on the learning of order information is a function of interitem contiguity; and (3) to test whether the total number of decoding operations or the total number of inhibitory connections is a better predictor of serial order learning.

\section{METHOD}

\section{Design}

One variable in the study was the consistency of the set of permutations learned by subjects. The set either defined an organizational system in which elements were assigned to a given node or it did not. It should be noted that the organization system was induced by the set of permutations and was not explicitly presented for study. The second variable tested was the structure of the system. Three structures were studied: balanced, right imbalanced, and left imbalanced. Balance is defined by the symmetry of the hierarchical arrangement of codes in a system. When the highest level division of codes represents an equal number of elements in each code, the system is balanced. Otherwise, the system is imbalanced and the direction of imbalance is defined by the position of the code representing more elements. Six groups of subjects were tested in a 2 by 3 design determined by the orthogonal combination of the consistency and balance factors. The six groups were as follows: (1) consistent, balanced; (2) consistent, left imbalanced; (3) consistent, right imbalanced; (4) inconsistent, balanced; (5) inconsistent, left imbalanced; and (6) inconsistent, right imbalanced.

At each level of balance, the consistent and inconsistent sets of permutations were matched for the number of times two letters occurred in the same order as in the base sequence. The sets of permutations for the hypothetical sequence ABCDEFGH and the frequencies with which letter pairs occurred adjacently are listed in Table 2 . The hierarchical organizations defined by the consistent permutation sets are shown in Figure 1. The number of decoding operations summed across transitions in those structures were: balanced, 22; right imbalanced, 25; and left imbalanced, 25 . In each case, the number of associative connections was 14 and the number of inhibitory connections was seven. It should be noted that degrees of balance, number of associative and inhibitory connections, and number of decoding operations pertain only to the consistent sets of permutations. The main reason for including the inconsistent sets was to determine the role of both the assignment of elements to unique positions within a hierarchy and the frequency with which letter pairs occur adjacently.

\section{Materials}

Two eight-consonant strings were used as base sequences. Each string was used in all six groups tested, so that 12 lists were used in all. The base sequences were GQSFPZCN and BXKDHWJM. No primary or secondary associate (Underwood \& Schulz, 1960) followed a given letter in either sequence.

The stimuli and responses were presented as slides. Stimulus slides consisted of a base sequence and a typewriter symbol. The symbols used were $\%, \$, \#, \&, *$, $@$, and ?. The study slides consisted of the base sequence, the symbol cue, and a particular 
Table 2A

Permutation Sets and Letter-Pair Frequencies for Hypothetical Sequence ABCDEFGH

\begin{tabular}{|c|c|c|c|c|c|}
\hline \multicolumn{2}{|c|}{ Right Imbalanced } & \multicolumn{2}{|c|}{ Balanced } & \multicolumn{2}{|c|}{ Left Imbalanced } \\
\hline Consistent & Inconsistent & Consistent & Inconsistent & Consistent & Inconsistent \\
\hline DEFGHABC & CDABEFGH & EFGHABCD & ABCDHEFG & FGHABCDE & ABDEFGHC \\
\hline CABDEFGH & ABDEFCGH & CDABEFGH & DABCEFGH & CDEABFGH & ABFGHCDE \\
\hline ABCGHDEF & ABCGHDEF & ABCDGHEF & ABEFCDGH & ABCDEGHF & ABCFDEGH \\
\hline BACDEFGH & DEFABCGH & BACDEFGH & EFABCDGH & BACDEFGH & CDEABFGH \\
\hline ABCEFDGH & ABCEFGDH & ABDCEFGH & CDEFABGH & ABDECFGH & ABCDEGFH \\
\hline ABCDFEGH & GHABCDEF & ABCDFEGH & ABEFGHCD & ABCEDFGH & BCDEFGHA \\
\hline ABCDEFHG & BCDEAFGH & ABCDEFGH & BCDEAFCH & ABCDEFHG & ABCDFGHE \\
\hline
\end{tabular}

Table 2B

Letter-to-Letter Frequencies

\begin{tabular}{cccc}
\hline & $\begin{array}{c}\text { Right } \\
\text { Imbalanced }\end{array}$ & Balanced & $\begin{array}{c}\text { Left } \\
\text { Imbalanced }\end{array}$ \\
\hline AB & 6 & 6 & 6 \\
BC & 5 & 4 & 4 \\
CD & 3 & 6 & 5 \\
DE & 5 & 2 & 6 \\
EF & 6 & 6 & 2 \\
FG & 3 & 4 & 5 \\
GH & 6 & 6 & 6 \\
\hline
\end{tabular}

permutation of the base sequence. Seven permutations were used for each base sequence and the symbols were randomly assigned to the various permutations.

\section{Procedure}

Subjects were tested individually in an anticipation paired associate task. When a stimulus (base sequence plus symbol) was presented, the subject was to call out from left to right the response (permutation) he anticipated seeing in the study slide. Subjects were allowed $10 \mathrm{sec}$ to respond. After the $10 \mathrm{sec}$ had elapsed, the st udy slide was presented for $10 \mathrm{sec}$.

The slides were projected by means of a Sawyer Rotomatic 707 projector onto a wall $1.5 \mathrm{~m}$ in front of the subjects. As the subject called out the response, the experimenter wrote down the response in a prepared booklet. A trial consisted of anticipating and studying the seven permutations of the stimulus sequence. Three random orderings of the stimulus-response pairs were used and each subject was tested for 10 trials

\section{Subjects}

The subjects were 72 introductory psychology students who appeared for testing in partial fulfillment of a course requirement. Twelve subjects were tested in each experimental condition and were assigned to the conditions randomly with the restriction that no condition had been tested with $n+1$ subjects before any other condition had been tested with n subjects.

\section{RESULTS}

\section{Permutation Learning}

The number of permutations correctly anticipated did not vary as a function of the letter set used. The total number of correct responses for the sequences GQSFPZCN and BXKDHWJM was 876 and 888 , respectively $[\mathrm{t}(34)=.14, \mathrm{p}>.05]$. Therefore, permutation learning scores were pooled across the two sequences.

The mean number of permutations correctly anticipated in each condition is presented in Table 3 . The data were subjected to a 2 by 3 analysis of variance, which showed that consistent permutations were easier to learn than inconsistent permutations $[\mathrm{F}(1,66)=66.18$, $\mathrm{MSe}=148.78, \mathrm{p}<.001]$. While degree of balance was not a significant factor $[F(2,66)=2.50, p>.05]$, the Consistency by Balance interaction was significant $[F(2,66)=3.70, p<.05]$. This interaction, however, is not easily interpreted in that the degrees of balance could not be assigned to the inconsistent sets which did not define unique hierarchical structures.

Since the level of balance is meaningful for only the consistent sets, a separate analysis was carried out for those three groups and the effect of balance was significant $[\mathrm{F}(2,33)=4.48, \mathrm{MSe}=138.6, \mathrm{p}<.02]$. An orthogonal comparison of the means showed that the right-branching and left-branching sets did not differ $[F(1,33)=1.24, p>.05]$, while the mean of those two sets was significantly different from that of the balanced set $[F(1,33)=7.77, p<.01]$.

\section{TEP Analysis}

The TEP scores were computed for each subject as follows. The number of times a given letter was incorrectly recalled was tallied for each position in the sequences and that score was divided by the number of times the preceding letter was recalled correctly. The TEP scores were calculated for each transition where a transition is identified by the position of the correct letter involved in computing the TEP score. The mean TEP scores are shown in Table 1 along with the mean number of decoding operations across permutations for that transition. The number of decoding operations for the inconsistent sets are those determined for the corresponding consistent sets.

Correlational analyses were carried out on the TEP scores and decoding operations for the six conditions.

Table 3

Mean Number of Correct Anticipations in Permutation Learning

\begin{tabular}{llcrrr} 
& \multicolumn{2}{c}{ Consistent } & & \multicolumn{2}{c}{ Inconsistent } \\
\cline { 2 - 3 } \cline { 5 - 6 } Condition & Mean & SD & & Mean & \multicolumn{1}{c}{ SD } \\
\hline Balanced & 43.7 & 11.63 & 13.66 & 14.58 \\
Right Branching & 34.9 & 11.84 & 7.16 & 9.52 \\
Left Branching & 29.5 & 11.84 & 17.25 & 13.12 \\
Mean & 36.03 & & 12.69 & \\
\hline
\end{tabular}


The $\mathrm{r}$ values for each analysis are shown in Table 1 , along with the $t$ and $p$ values. The analyses showed that for the consistent sets, the decoding operation values accounted for $84 \%$ of the variance in the TEP values. For the inconsistent sets, however, decoding operations accounted for only $40 \%$ of the TEP score variance. The significant relationship between decoding operations and TEP scores for the inconsistent-imbalanced set is puzzling, since the decoding operation metric should not apply for the inconsistent sets.

\section{DISCUSSION}

Three questions were addressed in the study: (1) Is a response set that defines a unique organization learned more quickly than one that does not? (2) Is the effect of organizational structure mediated by interitem contiguity? (3) Is there a relationship between the type of organization learned and the rate of learning?

Hierarchical organization systems have been shown to facilitate long-term memory of item information (Bower, Clark, Lesgold, \& Winzenz, 1969; Nelson \& Smith, 1972). In the present study it was shown that the structure facilitates the learning of order information in that response sets that assigned items to unique subgroupings (the consistent sets) were learned more quickly than responses that were inconsistent in their definition of the subgroupings. The acquisition of the structures implicit in the consistent response sets was indexed by a TEP analysis. The TEP scores were generally higher at transitions between subgroupings than within subgroupings for all three structures studied, and the pattern of those values was correlated with the pattern of decoding operations within the structure.

The distribution of TEP scores could not be accounted for merely in terms of how often the two items at a transition occurred in a particular order across the permutations set. If the TEP scores were a function of interitem co-occurrence frequency, then the patterns for both the consistent and inconsistent sets should not have differed in their relationship to the decoding operation metric since both types of sets were equated for interitem contiguity. The decoding operation measure, however, accounted for more than twice as much TEP score variance in the consistent condition than in the inconsistent condition. These findings suggest that organizational learning is not merely the result of experiencing items in contiguous spatiotemporal relationships.

Although both the Estes (1972) and Johnson (1970) theories are compatible with the findings up to this point, they differ in their predictions about the relative complexity of the different structures. Estes (1972) suggested that the number of inhibitory connections in a structure might be related to the difficulty of learning order information, although he provided no test of that prediction. On the other hand, Johnson (1970) has used decoding operations to predict structural complexity and has shown that the probability of omitting a subgroup of elements is directly related to the number of operations required to decode the code representing that subgroup. The balanced structure in the current study has fewer operations than the two imbalanced structures, yet all three structures have 14 associative and 7 inhibitory connections. The finding that the balanced structure led to a faster rate of permutation learning than the other structures that did not differ suggests that the Johnson (1970) model may be more appropriate as a description of the hierarchical system subjects learned.

It is possible, however, that the Estes (1972) and Johnson (1970) models of order information representation may refer to different domains of cognition. Recall that Estes (1972) proposed that memory for order information accrues via rehearsal processes. Rehearsal may be of such a nature as to preclude the formation of the types of structures sampled in this study. Specifically, all of the examples provided by Estes (1972, p. 184). yield structures in which the number of control elements at each level increases from top to bottom in the hierarchy. Yet the imbalanced structures in the present study had only one control element at the second lowest level. Although rehearsal may not produce such complex structures, these structures may be described in terms of the Estes model (Estes, Note 1). Rehearsal of particular segments of the sequence might have led subjects to become aware of only item-to-item contiguities, information that did not distinguish consistent and inconsistent sets. Since the consistent sets were learned more quickly, the rehearsal strategy apparently was not used.

The strategy subjects may have used was to learn what groups of letters were permutatable. That strategy would facilitate learning when a system generated by the permutations limits the possibilities. In this sense, then, the hierarchical organizations in the present study were learned implicitly (Reber, 1976). It may be that the Estes (1972) model describes the representation of order information when groupings are provided explicitly for subjects or when the groupings are generated through rehearsal. The Johnson (1970) model may apply to those cases as well as to the implicit learning situation tested in the present study.

\section{REFERENCE NOTE}

1. Estes, W. K. Personal communication, 1975.

\section{REFERENCES}

Bower, G. H. Organization factors in memory. Cognitive Psychology, 1970, 1, 18-46.

Bower. G. H., Clark, M. D., Lesgold, A. M., \& Winzenz, D. Hierarchical retrieval schemes in recall of categorized word lists. Journal of Verbal Learning and Verbal Behavior. 1969, 8. 323-343. 
Bower. G. H.. d Winzenz. D. Group structure, coding, and memory for digit series. Joumal of Experimental Psychology. Monograph Supplement. 1969. 802. Part 2), 1-17.

Estes. W. K. An associative basis for coding and organization in memory. In A. W. Melton \& E. Martin (Eds.), Coding processes in human memony. Washington. D.C: V. H. Winston. 1972.

HeALy. A. F. Separating item from order information in short-term memory. Journal of Verbal Learning and Verbal Behavior. 1974, 13, 644-655.

Johnson. N. F. The role of chunking and organization in the process of recall. In G. H. Bower (Ed.). The psychology of learning and motivation (Vol, 4). New York: Academic Press. 1970.

Johnson, N. F., \& Migdol. . D. M. Transfer and retroaction under conditions of changed organization. Cognitive Psychology, 1971, 2, 229-237.

Kenney, T. J. Permutation transformations on phrase structures in letter sequences. Journal of Experimental Psychology, 1969, 82, 28-33.
LASHLEY. K. S. The problem of serial order in behavior. In L. A. Jettress (Ed.). Cerebral mechanisms in behavior. New York: Wiley, 1951 .

Martin, E., \& Noreen, D. L. Serial learning: Identification of subjective subsequences. Cognitive Psychology, 1974, 6. 421.435

Nelson, T. O.. \& S Sith, E. E. Acquisition and forgetting of hierarchically organized information in long-term memory. Journal of Experimental Psychology, 1972, 95, 388-396.

REBER. A. S. Implicit learning of synthetic languages: The role of instructional set. Joumal of Experimental Psychology: Human Learning and Memory, 1976, 2. 88-94.

Underwood, B. J., \& Schulz, R. W. Meaningfulness and 'erbal learning. Philadelphia: Lippincott, 1960.

(Received for publication November 23, 1977; accepted January 16, 1978.) 